\title{
Periodic orbit quantization of the Sinai billiard in the small scatterer limit
}

\author{
Per Dahlqvist \\ Royal Institute of Technology, \\ S-10044 Stockholm, Sweden \\ and \\ Gábor Vattay \\ Eötvös University Budapest, \\ Department of Solid State Physics, \\ H-1088 Budapest, Múzeum krt. 6-8, Hungary
}

\begin{abstract}
We consider the semiclassical quantization of the Sinai billiard for disk radii $R$ small compared to the wave length $2 \pi / k$. Via the application of the periodic orbit theory of diffraction we derive the semiclassical spectral determinant. The limitations of the derived determinant are studied by comparing it to the exact KKR determinant, which we generalize here for the $A_{1}$ subspace. With the help of the Ewald resummation method developed for the full KKR determinant we transfer the complex diffractive determinant to a real form. The real zeros of the determinant are the quantum eigenvalues in semiclassical approximation. The essential parameter is the strength of the scatterer $c=J_{0}(k R) / Y_{0}(k R)$. Surprisingly, this can take any value between $-\infty$ and $+\infty$ within the range of validity of the diffractive approximation $k R \ll 4$, causing strong perturbation in spite of the smallness of the disk. We study the statistics exhibited by spectra for fixed values of $c$. It is Poissonian for $c= \pm \infty$, provided the disk is placed inside a rectangle whose sides obeys some constraints. For $c=0$ we find a good agreement of the level spacing distribution with GOE, whereas the form factor and two-point correlation function are similar but exhibit larger deviations. By varying the parameter $c$ from 0 to $\pm \infty$ the level statistics interpolates smoothly between these limiting cases. Any transitional level statistics can thus be found in the spectrum of a Sinai billiard with sufficiently small $R$ while we go from the quantum to the semiclassical limit $k \rightarrow \infty$.
\end{abstract}




\section{Introduction}

One of the fundamental questions of quantum chaos and mesoscopic physics is the relation between the classical motion and the energy levels of the quantum counterpart of the system [1, 2, 3n. Correlation of energy levels in chaotic systems in the semiclassical limit $(\hbar \rightarrow 0)$ are expected to coincide with those in Gaussian Random Matrix Ensembles (RME) with different symmetries (GOE, GUE etc.), while generic integrable systems are characterized by Poissonian level statistics [12]. Recently significant progress has been made concerning the applicability of RME for disordered and chaotic systems [4]. In disordered systems the two point correlation function has been expressed in terms of the spectral determinant of the diffusion equation. These results have been derived with supersymmetric techniques. It has been suggested that this result can be generalized to chaotic systems [4, 5], where the spectral determinant of the Perron-Frobenius operator plays a similar role.

Similar results have been derived with the help of semiclassical Gutzwiller type periodic orbit expressions [6]. This approach relies heavily on the so-called diagonal approximation for $t<t^{*}$, where $t^{*}$ is of the order of the Heisenberg time (see below), in order to, in an ingenious way, make statements about $t>t^{*}$. The suggested results of [4, 5] then follows after some further approximations.

One of the conclusions of these studies is that deviations from RME behavior is expected only when the chaotic mixing is slow and the characteristic time of mixing $T_{m}$ is comparable with the Heisenberg time. In a usual system the mixing time $T_{m}$ is a classical quantity characterizing the decay of classical correlation functions and as such it is of order $\hbar^{0}$. The Heisenberg time is the uncertainty time $T_{H} \sim \hbar / \Delta$ corresponding to the mean level spacing $(\Delta)$. In the semiclassical limit $\hbar \rightarrow 0$ the mean level spacing behaves like $\Delta \sim \hbar^{d}$, where $d$ is the dimension of the system and the Heisenberg time gets much larger than the mixing time.

Slow mixing can be expected if we perturb an integrable system with a small point like object, whose classical size can be neglected. In this case the only source of mixing is the quantum mechanical diffraction of waves on the small object. The diffraction constant governing the speed of the mixing is proportional with $\hbar^{2}$ and the resulting mixing time is proportional with $1 / \hbar$. In a two dimensional system the level spacing is also proportional with $\hbar^{2}$ and the ratio of the mixing time and the Heisenberg time remains fixed in the semiclassical limit $\hbar \rightarrow 0$. This gives the possibility to study systems, where corrections to RME remain finite. In this paper we study the Sinai billiard with a small scatterer, which, as we show here, is a strongly diffractive system and its level statistics deviates strongly from the RME result.

We are considering the standard Sinai billiard, a unit square with a reflective circular disk in the middle. We then develop the periodic orbit description of the Sinai billiard with small scatterer based on the diffractive extension[7] of the Gutzwiller 
trace formula. This is done in sections 2 and 3.1. The diffractive determinant we derive from the trace formula is directly related to the KKR determinant of Berry, which is discussed in section 3.2. We will study a straightforward generalization of Berry's method to the $A_{1}$ subspace, which exhibit some very interesting features, even in the diffractive or small radius limit. In section 4 we study the level statistics exhibited by the zeroes of the diffractive determinant for various values of the parameter $k R$. We then change from a square to a suitably chosen rectangle shape, in order to avoid the non generic degeneracies of the square billiard.

\section{Geometric theory of diffraction}

In this section we review the main steps of the derivation of the diffractive trace formula and spectral determinant introduced in Ref.[7]. The emphasis is on pointlike diffractive sources with no angular dependence in their associated diffraction constants. These point sources are placed inside a bounded and otherwise non diffractive system.

\subsection{Green functions}

For the description of diffraction effects in the semiclassical limit $(\hbar \rightarrow 0)$ Keller 8 introduced the Geometric Theory of Diffraction (GTD). According to this theory, the energy domain Green function can be divided into a geometric $(G)$ and a diffractive $(D)$ part:

$$
G\left(q, q^{\prime}, E\right)=G_{G}\left(q, q^{\prime}, E\right)+G_{D}\left(q, q^{\prime}, E\right),
$$

where the geometric part is the semiclassical Green function. The semiclassical Green function is a sum for all classical trajectories $(j)$ with energy $E$ connecting the starting point $q$ and the end point $q^{\prime}$

$$
G_{G}\left(q, q^{\prime}, E\right)=\sum_{j \in q \rightarrow q^{\prime}} G_{0}^{(j)}\left(q, q^{\prime}, E\right)
$$

where $G_{0}^{(j)}\left(q, q^{\prime}, E\right)$ is the Van Vleck-Gutzwiller Green function. It can be calculated from the action $S_{j}\left(q, q^{\prime}, E\right)=\int_{q}^{q^{\prime}} p d q$ calculated along trajectory $j$

$$
G_{0}^{(j)}\left(q, q^{\prime}, E\right)=A_{j}\left(q, q^{\prime}, E\right) e^{\frac{i}{\hbar} S_{j}\left(q, q^{\prime}, E\right)},
$$

where the amplitude $A_{j}\left(q, q^{\prime}, E\right)$ is the Van Vleck-Gutzwiller determinant [9] multiplied with the Maslov phase.

The diffractive part of the semiclassical Green function $G_{D}\left(q, q^{\prime}, E\right)$ describes

the effect of wave scattering on points $q_{k}^{s}$ being singular points of the classical 
dynamics [0, 10]. It is a sum over all possible trajectories which start from $q$, go to one of the singular points $q_{k}^{s}$, then leave the singular point and reach the end point $q^{\prime}$; or go and visit any combination of singular points before reaching the end point. A trajectory going trough one singularity contributes to the Green function with 8

$$
G_{0}^{(j)}\left(q, q_{i}^{s}, E\right) d_{k}\left(p, p^{\prime}, E\right) G_{0}^{\left(j^{\prime}\right)}\left(q_{i}^{s}, q^{\prime}, E\right)
$$

The generalization to multi scattered trajectories is straightforward, and will be developed in the next section. In eq (田), $j$ and $j^{\prime}$ are indices of the incoming and the outgoing classical trajectory, $d_{k}\left(p, p^{\prime}, E\right)$ is the diffraction constant associated with the singularity located at $q_{k}^{s}$. It depends on the direction of incoming and outgoing momenta $p$ and $p^{\prime}$ and energy $E$. The diffraction constant is the leading part of the exact quantum mechanical scattering amplitude of the singularity.

\subsection{Traces and periodic orbits}

Based on the expression for the Green functions in the previous subsection we can derive the diffractive version of the Gutzwiller trace formula [7].

The trace of the total Green function is a sum of its geometric and diffractive parts:

$$
\operatorname{Tr} G(E)=\operatorname{Tr} \tilde{G}_{G}(E)+\operatorname{Tr} G_{D}(E)+g_{0}(E)
$$

where the geometric part $\operatorname{Tr} \tilde{G}_{G}(E)$ is just the usual Gutzwiller trace formula, a sum over unstable periodic orbits possibly amended by a sum over neutral orbits and the contribution from zero length orbits is in $g_{0}(E) . \tilde{G}_{G}(0,0, E)$ is essentially the Green function $G_{G}(0,0, E)$ without the singular contribution coming from the zero length orbit. We concentrate here on the new term

$$
\operatorname{Tr} G_{D}(E)=\int G_{D}(q, q, E) d q
$$

To be more specific, we assume that the diffraction is caused by the presence of $N$ small disks located at $q_{k}^{s}$, where $1 \leq k \leq N$, whose diffraction constants $d_{k}(E)$ do not depend on the scattering angle 11. This will simplify the formulation considerably. We can easily see that the contribution to the Green function from all orbits via the disks $k_{1}$ and $k_{2}$

$$
\begin{aligned}
& \sum_{j_{0} ; q \mapsto q_{k_{1}}} G_{0}^{\left(j_{0}\right)}\left(q, q_{k_{1}}^{s}\right) d_{k_{1}} \sum_{j_{1} ; q_{k_{1}} \mapsto q_{k_{2}}} G_{0}^{\left(j_{1}\right)}\left(q_{k_{1}}^{s}, q_{k_{2}}^{s}\right) d_{k_{2}} \ldots \sum_{j_{2}: q_{k_{2}} \mapsto q^{\prime}} G_{0}^{\left(j_{2}\right)}\left(q_{k_{2}}^{s}, q^{\prime}\right) \\
& \quad=\sum_{j_{0} ; q \mapsto q_{k_{1}}} G_{0}^{\left(j_{0}\right)}\left(q, q_{k_{1}}^{s}\right) d_{k_{1}} \tilde{G}_{G}\left(q_{k_{1}}^{s}, q_{k_{2}}^{s}\right) d_{k_{2}} \ldots \sum_{j_{2}: q_{k_{2}} \mapsto q^{\prime}} G_{0}^{\left(j_{2}\right)}\left(q_{k_{2}}^{s}, q^{\prime}\right) .
\end{aligned}
$$

The generalization to multi scattered trajectories is now obvious. 
We can introduce a symbolic dynamics by simply labeling the disks by the corresponding indices $k$. In an open N-disk system a periodic disk sequence $\overline{k_{1} k_{2} \ldots k_{n}}$ codes the single periodic orbit visiting the disks in the prescribed order. In a bounded system it denotes all such periodic orbits e.g. the transition $\ldots k_{i} k_{i+1} \ldots$ denotes all (topologically distinct) ways to go from disk $k_{i}$ to disk $k_{i+1}$. In the following we will refer to periodic symbol codes and symbolic periodic orbits to avoid confusion with periodic orbits.

The calculation of the trace of the Green function $G_{D}\left(q, q^{\prime}, E\right)$ amounts to add up contributions from all distinct paths from $q$ to $q^{\prime}$ via $n \geq 1$ disk scatterings and then taking the trace. The basic building block $\tilde{G}_{G}\left(k_{i}, k_{i+1}, E\right)$ in semiclassical approximation is a sum over all paths from (see (2)).

Consider now a situation where the starting and ending points coincide $q=q^{\prime}$. The contribution from the symbolic path $k_{1}, k_{2} \ldots k_{n}$ to the trace is

$$
\int d q \sum_{j_{0} ; q \mapsto q_{k_{1}}} G_{0}^{\left(j_{0}\right)}\left(q, q_{k_{1}}^{s}\right) d_{k_{1}} \tilde{G}_{G}\left(q_{k_{1}}^{s}, q_{k_{2}}^{s}\right) d_{k_{2}} \cdots \tilde{G}_{G}\left(q_{k_{n-1}}^{s}, q_{k_{n}}^{s}\right) d_{k_{n}} \sum_{j_{n}: q_{k_{n}} \mapsto q} G_{0}^{\left(j_{n}\right)}\left(q_{k_{n}}^{s}, q\right)
$$

The integral over $q$ can be performed in the $\hbar \rightarrow 0$ limit by using the saddle point method. The result is

$$
\begin{gathered}
\frac{1}{i \hbar} \sum_{j: q_{k_{n}} \mapsto q_{k_{1}}}\left(T^{(j)}\left(q_{n}^{s}, q_{1}^{s}\right) G_{0}^{(j)}\left(q_{n}^{s}, q_{1}^{s}\right)\right) \\
\cdot \tilde{G}_{G}\left(q_{q_{k_{1}}}^{s}, q_{q_{k_{2}}}^{s}\right) d_{k_{1}} \tilde{G}_{G}\left(q_{k_{2}}^{s}, q_{k_{3}}^{s}\right) d_{k_{2}} \cdots \tilde{G}_{G}\left(q_{q_{n-1}}^{s}, q_{k_{n}}^{s}\right) d_{k_{n}},
\end{gathered}
$$

The stationary phase conditions select out all points $q$ lying on a trajectory between disk $k_{n}$ and $k_{1}$, that is, it selects periodic orbits.

To get the full contribution from the symbolic periodic orbit $\overline{k_{1} k_{2} \ldots k_{n}}$ one must add the contribution from all nonidentical cyclic permutations of the sequence $k_{1} k_{2} \ldots k_{n}$ in eqs. (8) and (9). The symbolic periodic orbit $\overline{k_{1} k_{2} \ldots k_{n}}$ can be a repetition of a shorter primitive (symbolic) periodic orbit $\overline{k_{1} k_{2} \ldots k_{n}}=\overline{p^{r}}$. The length of the code $p$ is denoted $n_{p}=n / r$. Instead of restricting ourself to all non identical permutations we can take all cyclic permutations and then divide by $r$.

The result of this somewhat laborious exercise in combinatorics can be conveniently expressed in terms of the derivative of the quantity

$$
t_{p}=\prod_{j=1}^{n_{p}} d_{k_{j}} \tilde{G}_{G}\left(q_{k_{j}}, q_{k_{j+1}}\right)
$$

as

$$
\operatorname{Tr} G_{D}=\sum_{p \in \Omega_{D}} \sum_{r=1}^{\infty} \frac{1}{r} \frac{d}{d E} t_{p}^{r}
$$


where $\Omega_{D}$ is the set of all primitive periodic symbol sequences taken from the alphabet $\{k ; 1 \leq k \leq N\}$ where $N$ is the number of singular points in the system.

We now introduce the spectral determinant $\Delta(E)$, defined through the logarithmic derivative

$$
\operatorname{Tr} G=-\frac{d}{d E} \log \Delta(E)
$$

Since the Green function is a sum (see Eq.5), the spectral determinant naturally factorizes as a product

$$
\Delta(E)=\Delta_{0}(E) \cdot \Delta_{G}(E) \cdot \Delta_{D}(E)
$$

where $\Delta_{0}(E)$ is associated with zero length orbits and corresponds to the mean level density, $\Delta_{G}(E)$ is the Gutzwiller-Voros zeta function associated with classical periodic orbits, possibly amended by neutral orbits. The diffractive determinant (or zeta function) now reads

$$
\Delta_{D}(E)=\prod_{p \in \Omega_{D}}\left(1-t_{p}\right)
$$

\section{Application to the Sinai billiard}

In this section we work out the diffractive spectral determinant of the Sinai billiard in the small disk limit. Then we show that the diffractive determinant is essentially the leading part of the Korringa-Kohn-Rostoker (KKR) determinant in the small disk limit.

\subsection{The diffractive spectral determinant}

The application for the Sinai billiard is now very simple, provided $k R$ is sufficiently small. The diffraction constant has been derived in Ref. [11, in the limit $k R \rightarrow 0$ it was found to be

$$
d(E) \sim-4 i \frac{J_{0}(k R)}{H_{0}^{(1)}(k R)} .
$$

It does not depend on the opening angle and the considerations in the previous section can be applied.

The billiard contains only a single disk and the symbolic dynamics introduced in the previous section has only a single letter implying the existence of one single primitive periodic code, and the resulting determinant is trivial

$$
\Delta_{D}(E)=1-d(E) \tilde{G}_{G}(0,0, E) .
$$

The geometry of the Sinai billiard is easy to visualize if one unfolds it into a quadratic lattice of disks. A trajectory from the diffractive object (i.e. the small 
disk) and back to itself is characterized by a lattice vector $\rho=\left(\rho_{x}, \rho_{y}\right)$ where $\rho_{x}$ and $\rho_{y}$ can take any integer value. The geometric Green function $G_{G}(0,0, E)$ may be expressed as a sum over lattice vectors

$$
\tilde{G}_{G}(0,0, E)=\sum_{\rho \neq(0,0)} G_{0}(0, \rho, E)
$$

with the zero length vector $\rho=(0,0)$ excluded. The Green function $G_{0}$ may be expressed in terms of (the semiclassical limit of) a free Green function

$$
\tilde{G}_{G}(0,0, E)=\sum_{\rho \neq(0,0)} G_{f r e e}(0, \rho, E)(-1)^{\rho_{x}+\rho_{y}},
$$

where $(-1)^{\rho_{x}+\rho_{y}}$ is a phase index associated with the reflection on the square wall. But in order to improve the approximation we use the exact free Green function in two dimensions

$$
G_{f r e e}\left(x, x^{\prime}\right)=-\frac{i}{4} H_{0}^{(1)}\left(k\left|x-x^{\prime}\right|\right) .
$$

The resulting expression is

$$
\Delta_{D}(E)=1-d(E) \sum_{\rho \neq(0,0)}\left(-\frac{i}{4}\right) H_{0}^{(1)}(k|\rho|)(-1)^{\rho_{x}+\rho_{y}}
$$

where $k=\sqrt{2 E}$ is the momentum in dimensionless units $\hbar=m=1$. The sum for the lattice vectors is the exact Green function of the empty square with the zero length term removed.

The Green function can also be expressed via the eigenfunctions of the empty square. The wave functions can be categorized according to the different irreducible representations of $C_{4 v}$. There are four one dimensional $\left(A_{1}, A_{2}, B_{1}\right.$ and $\left.B_{2}\right)$ representations and one two dimensional $(E)$. The disk is centered at the point where all the symmetry lines meet. Wave functions of the empty square billiard vanish at this point except for the $A_{1}$ subspace. Accordingly, only the energies belonging to this subspace are affected by diffraction in leading semiclassical order as will be demonstrated later.

\subsection{The KKR determinant}

The full quantum Sinai billiard problem can be solved with the KKR method introduced in this context by Berry in Ref.[12], where the $A_{2}$ subspace has been worked out in detail. The method leads to a determinant $D_{K K R}$ whose zeroes yield the eigenvalues of the problem. The generalization to other subspaces are fairly straightforward. Here, without repeating the derivation of Ref. [12], we quote the relevant results for the $A_{1}$ and $A_{2}$ subspaces and show that the periodic orbit expression (20) is essentially the leading part of the KKR determinant. 
The effective dimension of the KKR matrix is $\sim(k R) / 4$. For $k R<4$, which we call the diffractive zone, it is essentially one dimensional. The determinant is given by the leading element of the matrix

$$
\Delta_{K K R}(k)=\left\{\begin{array}{ll}
1+\frac{J_{0}(k R)}{Y_{0}(k R)} S_{0}^{r}(e) & \text { in the } A_{1} \text { subspace } \\
1+\frac{J_{4}(k R)}{Y_{4}(k R)}\left(S_{0}^{r}(e)-S_{8}^{r}(e)\right) & \text { in the } A_{2} \text { subspace }
\end{array},\right.
$$

where the real functions $S_{l}^{(r)}(e)$ are related to the structure constants

$$
S_{l}(e)=-i \sum_{\rho \neq \mathbf{0}} H_{l}^{(1)}(k \rho) e^{i l \phi_{\rho}} \cdot\left\{\begin{array}{ll}
(-1)^{\rho_{x}+\rho_{y}} & \text { in the } A_{1} \text { subspace } \\
1 & \text { in the } A_{2} \text { subspace }
\end{array}\right. \text {. }
$$

via

$$
S_{l}(e)=S_{l}^{r}(e)+i \delta_{0, l} .
$$

$e=(k / 2 \pi)^{2}$ is a rescaled energy (denoted by $E$ in [12]).

The structure constants, as they stand, do not converge. They can be evaluated by transforming them to sums over the dual lattice using the Ewald resummation method[12]. The resulting expressions are

$$
\begin{aligned}
& S_{l}^{(r)}(e) \approx \frac{1}{\pi^{2}} \sum_{\mathbf{v}}\left(\frac{\mathrm{v}^{2}}{e}\right)^{l / 2} \frac{\exp \left(l / 2\left[1-\mathrm{v}^{2} / e\right]\right)}{e-\mathrm{v}^{2}} l \neq 0 \\
& S_{0}^{(r)}(e) \approx \frac{1}{\pi^{2}} \sum_{\mathbf{v}} \frac{\exp \left(Q\left[1-\mathrm{v}^{2} / e\right]\right)}{e-\mathrm{v}^{2}}-E_{i}(Q) / \pi \quad l=0
\end{aligned}
$$

We have introduced the vector $\mathbf{v}$ in the dual lattice

$$
\begin{array}{ll}
\mathbf{v}=\left(v_{x}+\frac{1}{2}, v_{y}+\frac{1}{2}\right) & \text { in the } A_{1} \text { subspace } \\
\mathbf{v}=\left(v_{x}, v_{y}\right) & \text { in the } A_{2} \text { subspace } .
\end{array}
$$

where $v_{x}$ and $v_{y}$ are integers. The poles of the expressions located in $\mathrm{v}^{2}$ are the eigenvalues of the empty square billiard. The smaller the number $Q$ the more accurate the method works.

Combining Eqs. (15), (20), (21) and (22) we find the desired relation between the diffractive limit of the KKR determinant and the diffractive determinant

$$
\Delta_{K K R}(k)=\left(1-i \frac{J_{0}(k R)}{Y_{0}(k R)}\right) \Delta_{D}(k) .
$$

This relation is one of the main results of the paper.

The KKR determinant, and thus the diffractive spectral determinant, exhibit poles at the unperturbed eigenvalues $e_{\mathbf{v}}^{(0)}=\mathbf{v}^{2}$. They cancel the zeroes of the geometric determinant $\Delta_{G}(k)$ according to the formula

$$
\Delta(k) \sim \Delta_{G}(k) \Delta_{D}(k),
$$


and give rise to corrected eigenvalues given by the zeroes of $\Delta_{D}(k)$. This is an important property of diffractive zeta functions: they cancel the geometric zeros and create new ones.

Note that there is no contribution coming from isolated unstable orbits in our system, $\frac{d}{d E} \log \Delta_{G}(k)$ is just the Berry-Tabor sum for the square billiard [13.

\section{Investigation of the energy levels}

The investigation of the KKR determinant for all symmetry subspaces is not a superfluous exercise. Next we show that the $A_{1}$ subspace will exhibit some very interesting features, to which we devote the rest of the paper.

The quantum eigenvalues in the diffractive limit can be determined from the equation

$$
\Delta_{D}(k)=0 .
$$

or euivalently from the real equation

$$
S^{(r)}(e)+c(k R)=0
$$

where

$$
c(k R)=\frac{Y_{0}(k R)}{J_{0}(k R)}
$$

\subsection{Perturbation theory}

When $c(k R)$ is large one can use perturbation theory to compute the roots of Eq. (30) (see Ref.[12]). One thus assumes that the difference between a perturbed level $e$ and an unperturbed level $e^{(0)}$ is small. $S_{0}^{(r)}(e)$ close to $e^{(0)}$ may then be approximated by

$$
S_{0}^{(r)} \approx \frac{8}{\pi^{2}\left(e-e^{(0)}\right)}
$$

yielding the perturbed eigenvalue

$$
e \approx e^{(0)}-\frac{8}{\pi^{2}} \frac{J_{0}(k R)}{Y_{0}(k R)}
$$

Fig. 1 illustrates how well this expression works for $R=0.025$, where we have plotted the distance from an eigenvalue of the Sinai billiard $(R=0.025)$ and the nearest eigenvalue of the square billiard. The expression above applies only to non-degenerate states. For a $n$-degenerate unperturbed energy, $n-1$ levels remain almost unaffected, which is clearly seen on Fig. 1.

There are two regions in the range $0<k R<4$ where perturbation theory should work in principle. 
The first is the limit $k R \rightarrow 0$. Taking well known asymptotic expressions [14] for the Bessel functions one gets

$$
e=e^{(0)}-\frac{1}{2 \pi} \frac{1}{\log (k R / 2)+\gamma},
$$

where $\gamma=0.57721 \ldots$ is Euler's constant. However, this expression goes very slowly to zero as $k R \rightarrow 0$ and unless the radius is very small perturbation theory does not even apply to the ground state $e_{1}^{(0)}=0.5$.

The second is where $c(k R)$ has a pole within the diffractive zone $k R<4$, namely at $k R=j_{0,1}=2.40482 \ldots$. Close to this value, the perturbation expression works very well, as it can be seen on the scatter plot Fig. 1. Note, that the index of the levels is now shifted one step up relative to the unperturbed ones, this is why we chose to plot the nearest distance to an unperturbed eigenvalue. We note that $c(k R)$ exhibit a zero at $k R=y_{0,1} \approx 0.899$ so the perturbation theory also breaks down deep inside the diffractive zone.

As a comparison, in the $A_{2}$ subspace the perturbation is on average[12]

$$
<e-e_{0}>=-\frac{8}{\pi^{2}} \frac{J_{4}(k R)}{Y_{4}(k R)} \sim-\frac{1}{18 \pi}\left(\frac{k R}{2}\right)^{8} .
$$

Perturbation theory works well almost throughout the whole diffractive region. So the spectrum in the $A_{2}$ representation, in the diffractive zone, can hardly be expected to be particularly interesting.

For $A_{1}$, on the other hand, $|c(k R)|$ will range from 0 to $\infty$ in the interval $0<k R<y_{0,1} \approx 0.899$ which is well inside the diffractive zone.

\subsection{Level statistics in the diffractive limit}

In this section we solve Eq. (30) numerically and study the statistics of levels. The essential parameter of the problem is $k R$ since the mean level spacing $\Delta_{e}$ does not depend on energy $e$. Here we will study spectra for fixed values of $c(k R)$. The idea of keeping $c$ and thus $k R$ fixed needs some explanation. If we decrease $R$ and keep $k R$ fixed by increasing $k$, more and more levels are squeezed in a neighborhood of some $k=k_{0}$. We then can safely replace $k R$ by $k_{0} R$ and it is possible to obtain a sufficiently large number of levels around $k_{0}$ for which the value of $c$ is approximately constant and given by $c\left(k_{0} R\right)$. For obvious reasons we calculate the bottom part of the spectrum. However, this sample should exhibit the same statistics as any comparable sequence of levels, e.g. around $k_{0}$. The reason is that the location of a level depends on the parameter $k R$ and the location of the unperturbed levels in the environment and the statistics of these unperturbed levels is well defined and given by a Poissonian process. 
In the actual calculation we made a minor modification of the conventional Sinai billiard, since the empty square billiard has degeneracies in its spectrum. Their origin is number theoretical and is connected with the high degree of symmetry in the system. If a disk is present, these degeneracies are split by higher order terms in the KKR determinant. Within the scope of the present diffractive determinant they remain there. In the following we want to avoid this complication and replace the square by a rectangle with sides $a=1$ and $b=2^{1 / 4}$. The function $S_{0}^{(r)}(e)$ is modified accordingly

$$
S_{0}^{(r)}(e) \approx \frac{1}{a b} \frac{1}{\pi^{2}} \sum_{\mathbf{v}} \frac{\exp \left(Q\left[1-\mathrm{v}^{2} / e\right]\right)}{e-\mathrm{v}^{2}}-E_{i}(Q) / \pi,
$$

where $\mathbf{v}=\left(\left(v_{x}+1 / 2\right) / a,\left(v_{y}+1 / 2\right) / b\right)$ is now the dual lattice of the rectangle.

We investigate three statistical measures on the spectra. First we will investigate the integrated probability distribution of neighboring levels

$$
P(s)=\sum_{n} \theta\left(s-\left(e_{n+1}-e_{n}\right) / \Delta_{e}\right),
$$

where $\Delta_{e}$ is the mean level spacing around the energy $e$ and $\theta(x)$ is the step function. Then we investigate the two point correlation function of levels

$$
\begin{gathered}
R(\epsilon)=\left\langle\sum_{i j} \delta\left(\left(e-e_{i}\right) / \Delta_{e}+\epsilon / 2\right) \cdot \delta\left(\left(e-e_{j}\right) / \Delta_{e}-\epsilon / 2\right)\right\rangle_{e} \\
=\delta(\epsilon)+\left\langle\sum_{i \neq j} \delta\left(\left(e-e_{i}\right) / \Delta_{e}+\epsilon / 2\right) \cdot \delta\left(\left(e-e_{j}\right) / \Delta_{e}-\epsilon / 2\right)\right\rangle_{e} \equiv \delta(\epsilon)+\tilde{R}(\epsilon)
\end{gathered}
$$

where the average is taken for a large number of energies. We also compute its Fourier transform, the form factor,

$$
K(\tau)=\int_{-\infty}^{+\infty} e^{2 \pi i \tau \epsilon}(R(\epsilon)-1) d \epsilon
$$

The correlation function and the form factor are computed over a gaussian window centered at the middle of the sample spectrum, its width is about one sixth of the sample size. The results are then smeared with another gaussian. We choose the width of this gaussian rather small so some (non significant) oscillations due to finite statistics will remain in the plots.

In Fig. 2 we show results for $c=0$, with 5000 levels computed. The level spacing curve (Fig 2a) is close to the prediction of the random matrix theory (GOE), with a small but significant deviation. An exact agreement is hardly possible because the levels are locked between the unperturbed levels [12]. The close agreement with GOE is in this perspective rather surprising.

The formfactor $K(\tau)$ is plotted in Fig 2b. There is again good agreement with GOE except for small $\tau$. The bump for small values of $\tau$ indeed persist rather 
high up in the spectrum (of the full KKR determinant) and is quite similar to the behavior predicted in [15].

The correlation function $\tilde{R}(\epsilon)$ is plotted in Fig. 2c. The deviation from GOE is now most pronounced around $\epsilon \approx 1$.

In Fig. 3 we show the correlation function $\tilde{R}(\epsilon)$ for a geometric sequence of $c$ 's, each spectrum contains 1500 levels. We note how it, in a uniform way, interpolates between a Poissonian result and the GOE-like.

\section{Concluding Remarks}

The KKR determinant may be recast into a periodic orbit expressions in the limit $k R \rightarrow \infty$ [12]. Periodic orbit calculations are very laborious in this limit, it is questionable whether it provides a useful method of extracting (many) eigenvalues for bound chaotic systems [16].

What motivated us in this paper, is that the KKR determinant yields (diffractive) periodic orbit expressions also in the limit $k R \rightarrow 0$. This diffractive limit is very feasible form a periodic orbit point of view.

A traditional symbolic dynamics for the Sinai billiard uses the lattice vectors $\rho$ as symbols [17]. In the diffractive limit, this symbolic dynamics is complete. The reason for this is the absence of shadow behind disks in the $k R \rightarrow 0$ limit. Moreover, the diffraction constant is independent of the scattering angle. In the language of cycle expansions [18], these circumstances imply that the cycle expansion (20) is entirely given by the (well defined) fundamental part, the curvature correction vanish identically.

So, what we have presented here is a very straight forward application of the cycle expansion technique as well as of the geometric theory of diffraction. Yet, the results are non-trivial. Even if we have not used the diffractive determinant for computations as it stands (20) we have demonstrated that study of spectral statistics is in principle approachable for periodic orbit theories in this case.

A natural extension of this work is to increase the number of small scatterers and study whether GOE emerges as the number of scatterers tends to infinity and are distributed randomly, preliminary results indicate that this is indeed the result 19.

This work was supported by the Swedish Natural Science Research Council (NFR) under contract no. F-AA/FU 06420-312 and no. F-AA/FU 06420-311., the Hungarian Science Foundation OTKA (F019266/F17166/T17493) and the Hungarian Ministry of Culture and Education FKFP 0159/1997. G. V. thanks for the hospitality of the Mechanics Department of the Royal Institute of Technology during his stay. 


\section{References}

[1] O. Bohigas and M.-J. Giannoni, in Mathematical and Computational Methods in Nuclear Physics, ed.: J. Desha, J. M. Gomez and A. polls, Lecture Notes in Physics, Vol. 209 (Springer, Berlin 1984)

[2] B. Eckhardt, Phys. Rep. 163, 205 (1988)

[3] F. Haake, Quantum Signatures of Chaos (Springer, Berlin 1991)

[4] A. V. Andreev and B. L. Altshuler, Phys. Rev. Lett. 75, 902 (1995)

[5] O. Agam, A. V. Andreev and B. D. Simons, Chaos Solitons and Fractals, 8, 1099, (1997).

[6] E. B. Bogomolny and J. P. Keating, Phys. Rev. Lett. 77, 1472 (1996).

[7] G. Vattay, A. Wirzba and P. E. Rosenqvist, Phys. Rev. Lett. 73, 2304 (1994)

[8] J. B. Keller, J. Opt. Soc. Amer. 52116 (1962)

[9] M. C. Gutzwiller, Chaos in classical and quantum mechanics, Springer Verlag, New York (1990)

[10] H. Bruus and N. Whelan, Nonlinearity 9, 1023-1047 (1996)

[11] P. Rosenqvist, N. D. Whelan, A. Wirzba, J. Phys. A29, 5441 (1996)

[12] M. V. Berry, Ann. Phys. N.Y. 131, 163 (1981)

[13] M. V. Berry and M. Tabor, Proc. R. Soc. Lond. A 349, 101 (1974).

[14] M. Abramovitz and I. A. Stegun, Handbook of mathematical functions, Washington: National Bureau of Standards, (1964).

[15] P. Dahlqvist, Physica D 83, 124 (1995).

[16] P. Dahlqvist, Chaos Solitons and Fractals, 8, 1011, (1997).

[17] P. Dahlqvist, Nonlinearity 8, 11 (1995).

[18] R. Artuso, E. Aurell and P. Cvitanović, Nonlinearity 3, 325 and 361, (1990).

[19] P. Dahlqvist, From chaotic to disordered systems - a periodic orbit approach, in preparation. 


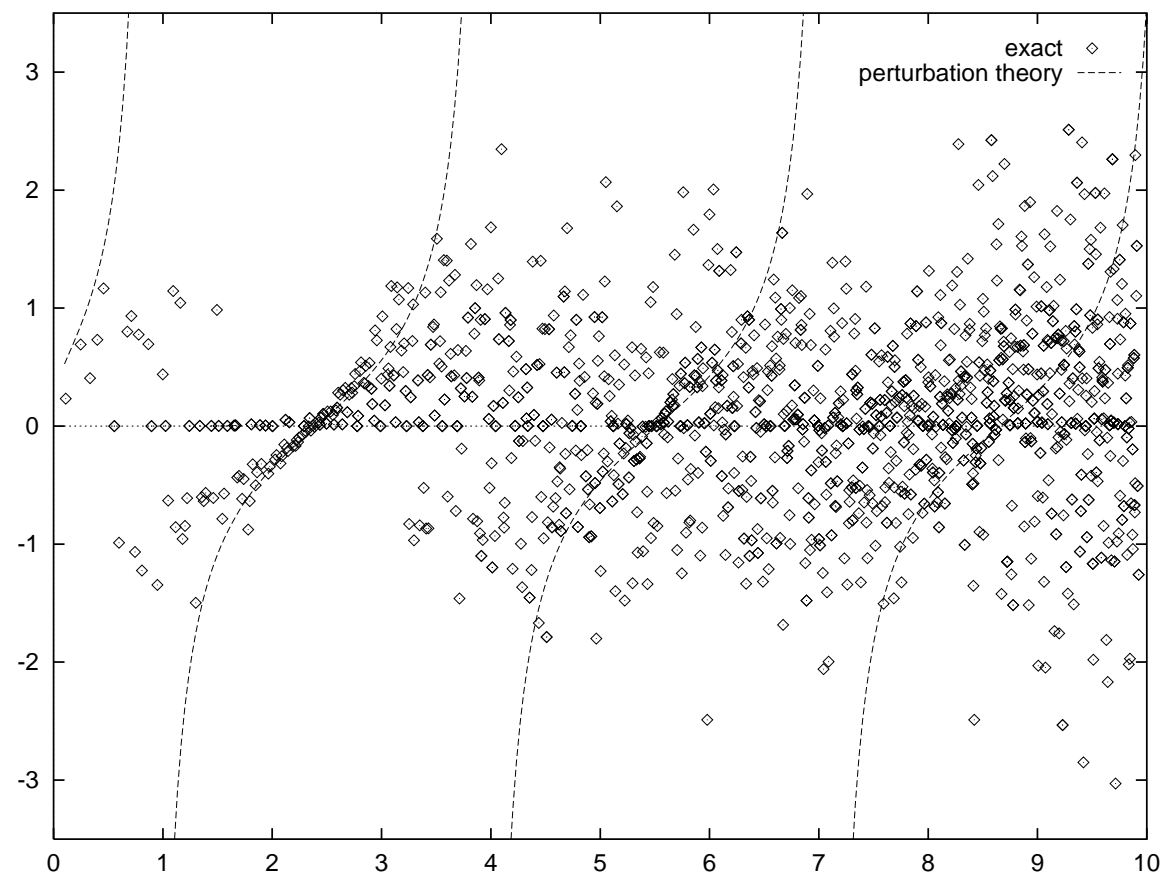

Figure 1: Distance between eigenvalues of the Sinai billiard and the nearest eignvalue of the empty square compared with perturbation theory. The eigenvalues are from the $A_{1}$ subspace for radius $R=0.025$. 

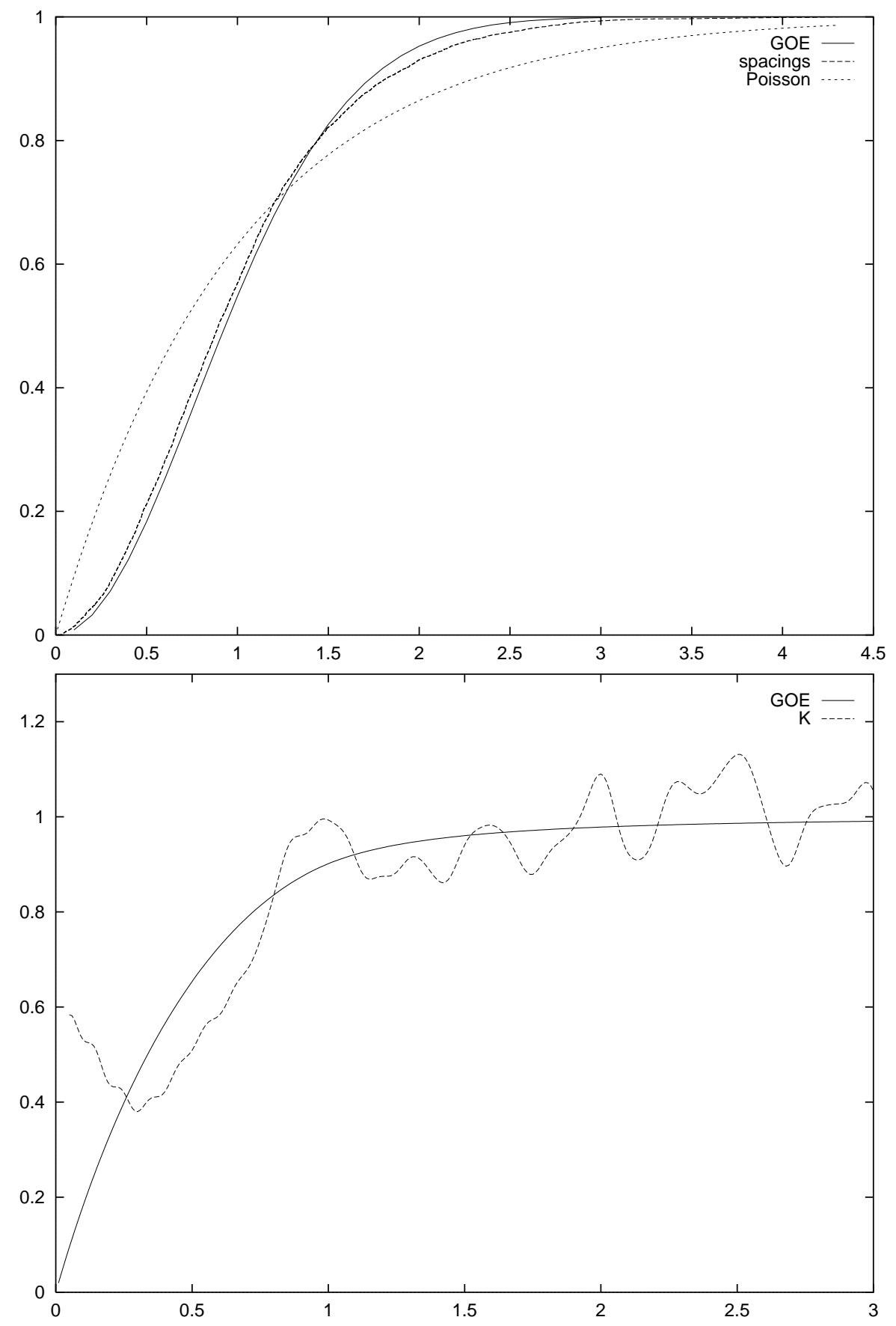


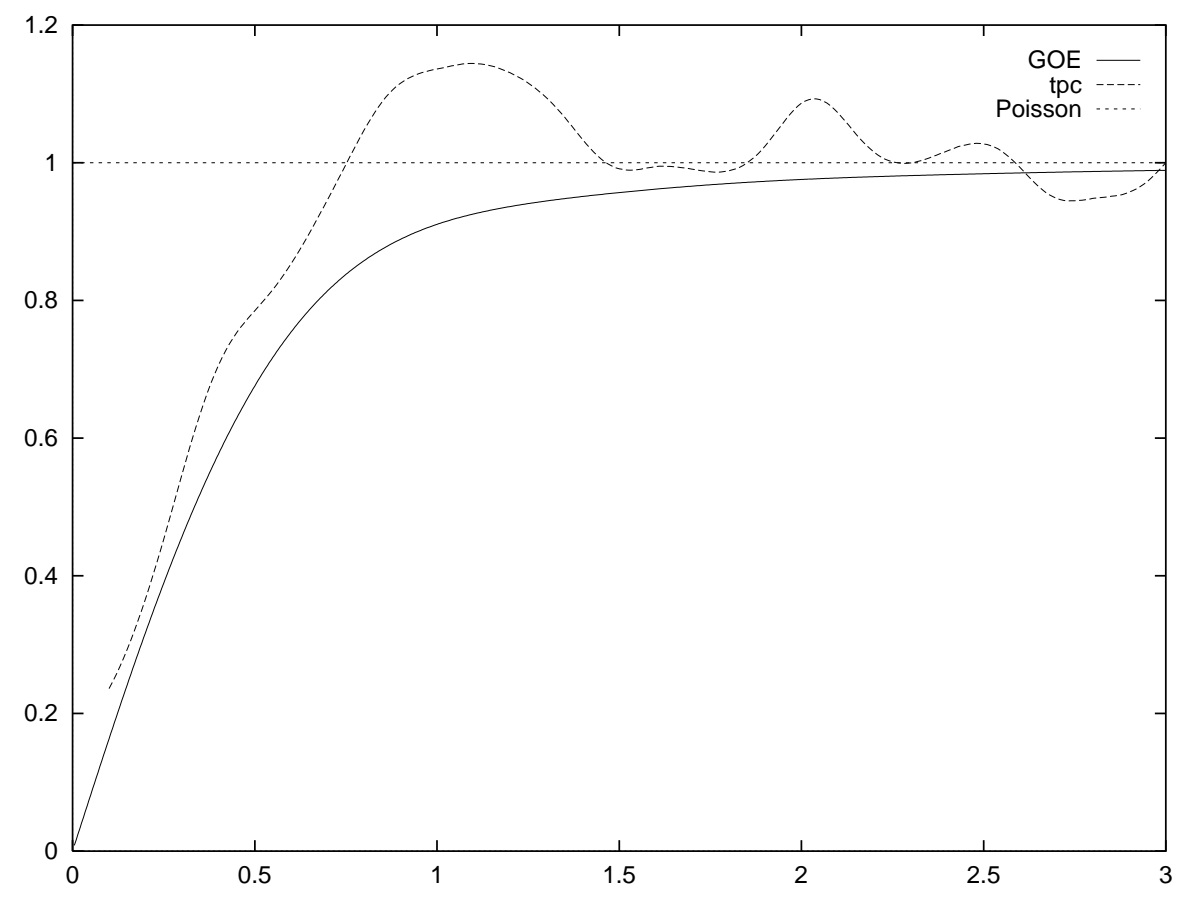

Figure 2: a) Integrated level spacing distribution. b) Form factor. c) Two point correlation function. Eigenvalues computed from eq (30) with $c=0$ for a rectangular Sinai billiard. 


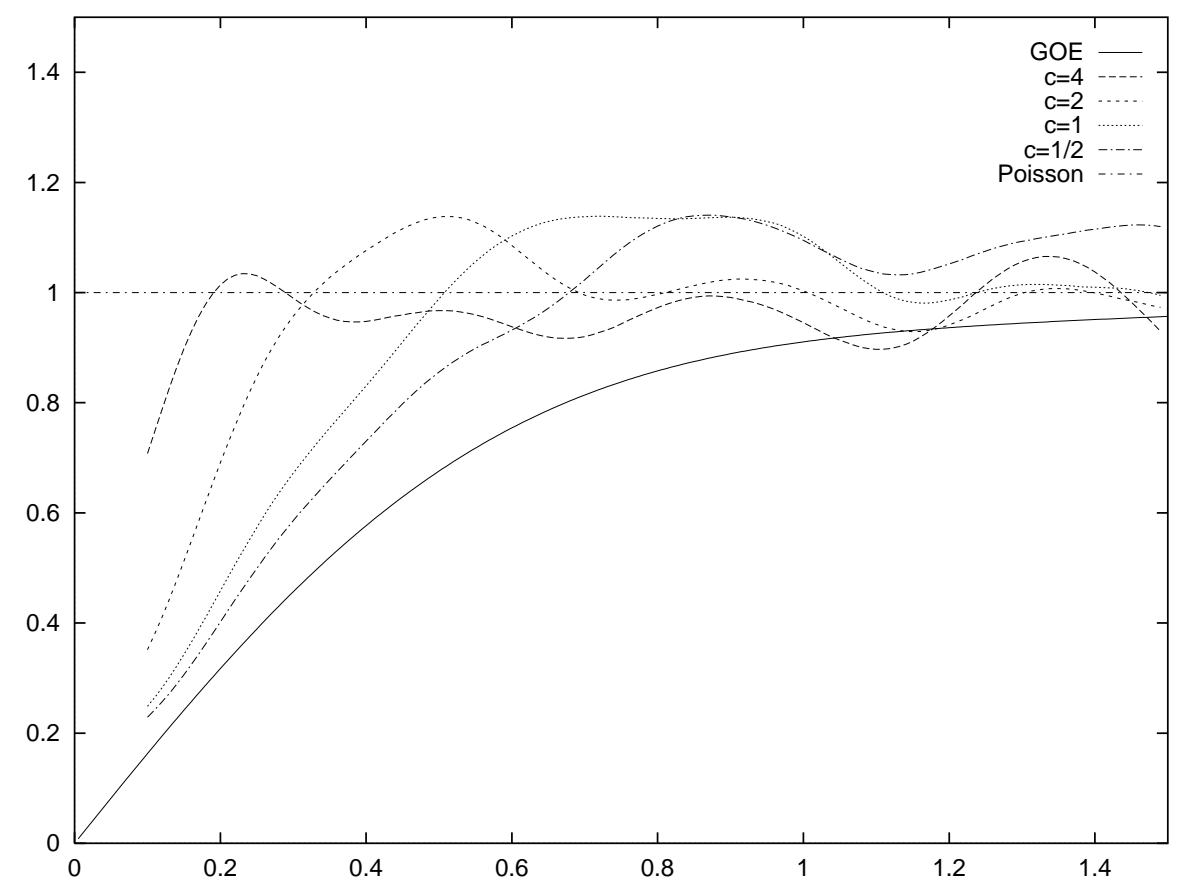

Figure 3: Two point correlation function from eq (30) for different values of $c$. 